\title{
Formación académica e iniciativa emprendedora de los estudiantes de educación superior tecnológica
}

\section{Academic training and entrepreneurial initiative of higher technological education students}

Yessica García-Hernández ', Jesús Alberto García-Rojas ${ }^{2}$, Ana Isabel Ramírez-Sabino ${ }^{3}$, María de Lourdes Amador-Martinez ${ }^{4}$

INFORMACIÓN DEL

\section{ARTÍCULO}

Fecha de recepción: 22 de Julio de 2020.

Fecha de aceptación: 21 de Noviembre de 2020

Doctorante en Ciencias EconómicoAdministrativas, Universidad Autónoma del Estado de Hidalgo. Docenteinvestigadora, Instituto Tecnológico Superior del Oriente del Estado de Hidalgo-México. E-mail: ygarcia@itesa.edu.mx

Código ORCID:

https://orcid.org/0000-0002-4482-7275

2 Doctor en Investigación Educativa, Universidad de Puebla. Docenteinvestigador, Instituto Tecnológico Superior del Occidente del Estado de Hidalgo-México. E-mail: jgarcia@itsoeh.edu.mx

Código ORCID:

https://orcid.org/0000-0002-0292-0789

3 Maestra en Ciencias de Administración, Colegio de Estudios de Posgrado de la Ciudad de México. Docente-investigador, Instituto Tecnológico Superior de Huichapan-México.

E-mail: airamireza@iteshu.edu.mx

Código ORCID

https://orcid.org/0000-0002-4478-9838

4 Doctorante en Ciencias de la Gestión Administrativa, Universidad Politécnica de Tulancingo. Docente-investigador, Instituto Tecnológico Superior del Oriente del Estado de Hidalgo-México.

E-mail: lamador@itesa.edu.mx

Código ORCID

https://orcid.org/0000-0001-8963-6264

CITACIÓN: García Hernández, Y., García Rojas, J.A., Ramírez Sabino, A.I., \& Amador Martínez, M.L. (2020). Formación académica e iniciativa emprendedora de los estudiantes de educación superior tecnológica. Podium, 38, 123-138. doi:10.31095/podium.2020.38.8

\section{ENLACE DOI:}

http://dx.doi.org/10.31095/podium.202 0.38 .8

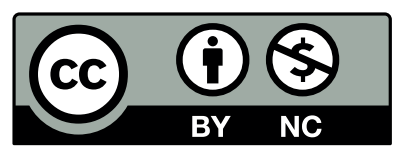

\section{Resumen}

El emprendimiento es un elemento clave para la generación de empleo, y las Instituciones de Educación Superior (IES) juegan un papel fundamental en la formación académica y fomento al desarrollo empresarial. El objetivo de este artículo es evaluar la iniciativa emprendedora de los estudiantes de educación superior tecnológica en México, mediante la Teoría del Comportamiento Planificado, con la finalidad de identificar diferencias significativas respecto a la formación académica. Se aplicó el cuestionario GUESSS (Global University Entrepreurial Spirit Student's Survey) a 919 estudiantes. Es un estudio empírico, cuantitativo, de corte transversal, con alcance descriptivo y explicativo. Se aplicó el método de análisis de varianza factorial ANOVA. Se comprueba la hipótesis que plantea la existencia de diferencias estadísticamente significativas respecto a la formación académica en las puntuaciones medias de actitud personal hacia el emprendimiento, normas subjetivas y el índice global de iniciativa emprendedora.

\section{Palabras Clave:}

Formación académica, iniciativa emprendedora, estudiantes, educación superior tecnológica, emprendimiento, GUESSS.

Clasificación JEL: I21, I23, M13.

\begin{abstract}
Entrepreneurship is a key element for job creation, and for Higher Education Institutions. It plays a fundamental role in academic training and it furthers business development. This article purports to evaluate higher technological education students' entrepreneurial initiative in Mexico. Planning Behavior Theory is used to identify significant differences in academic training. The GUESSS (Global University Entrepreneurial Spirit Student's Survey) questionnaire was applied to 919 students. The research was an empirical, quantitative, cross-sectional study with a descriptive and explanatory scope. The ANOVA analysis method of factorial variance was use. The hypothesis that concerns the existence of statistically significant differences regarding academic training was proved; it concerned medium personal attitude scores towards entrepreneurship, subjective rules, and entrepreneurial initiative global index.
\end{abstract}

Keywords:

Academic training, entrepreneurial initiative, students, higher technological education, entrepreneurship, GUESSS.

JEL Classification: I21, I23, M13.

$$
123
$$

PODIUM No. 38, Diciembre 2020, pp. 123-138

(C) Universidad Espíritu Santo - UEES

ISSN: 1390-5473 e-ISSN: 2588-0969 


\section{Introducción}

A través del tiempo, diversos estudios académicos y de organismos internacionales han establecido que la Educación Superior es el eje sobre el cual se sustenta el capital humano que integrará el sistema económico (OECD, 2019). El crecimiento económico de un país depende del número y calidad de sus empresas, ya que estas inciden en la generación de empleo, desarrollo regional e innovación de productos $\mathrm{y}$ servicios, por lo tanto, en primera instancia resulta fundamental estudiar a quienes las crean y asumen el riesgo de ser emprendedores (Bucardo, Saavedra y Camarena, 2015). Por lo anterior, desde hace algunos años, el tema del emprendimiento ha ocupado diversos espacios en la academia, investigación y en las políticas públicas, pues es indiscutible, la aportación a la dinámica económica que hoy en día generan los emprendedores, quienes constituyen la base de la pirámide empresarial, debido a que están en proceso de crear, desarrollar o consolidar una empresa (Secretaría de Economía, 2017).

Según Hernández y Sánchez (2017) existe una creciente preocupación por enfatizar el impacto del proceso educativo para generar emprendedores, debido a que los modelos educativos forman la esencia del emprendimiento y puede ser que en ocasiones el sistema educativo no genere las competencias para los futuros emprendedores. Por lo cual, las implicaciones para las Instituciones de Educación Superior (IES) se refieren a reconocer la importancia de la evaluación de los avances y retrocesos de los modelos educativos orientados a fomentar la cultura empresarial, el espíritu e iniciativa empresarial y que serán el impulso para los emprendedores jóvenes. Entonces, la educación en emprendimiento, debe utilizar enfoques innovadores para la enseñanza y el aprendizaje, asimismo, si se integra en el currículo y se usa como parte de la formación académica, podría llegar a todos los estudiantes y facilitar el desarrollo de una gran variedad de competencias transversales junto con la creación de conocimientos y habilidades emprendedoras, logrando así, la formación de egresados competitivos que cuenten con las competencias necesarias para su formación y desempeño profesional (Contreras-Velásquez, WilchesDuran, Graterol-Rivas y BautistaSandoval, 2017).

Una de las principales problemáticas a las que se enfrentan los jóvenes con estudios superiores, es que en la última década las condiciones de trabajo han empeorado, debido al uso ineficiente de sus competencias, por la informalidad y la sobre-cualificación (OECD, 2019). El desempleo y el subempleo juvenil son problemas generados por cuestiones sistémicas y ciclos económicos, que se han practicado desde hace tiempo, incluso antes de la actual crisis sanitaria, por lo cual el panorama en empleo juvenil no vislumbra un escenario optimista (De Domingo-Soler, Naveda-Jácome, RodríguezProaño, y Muñoz-Pumagualle, 2020).

Al respecto, Duval-Couetil, Gotch y Yi (2014) señalan que los estudiantes no 
tienen una sólida formación en materia de emprendimiento, pero desean recibir capacitación, para fortalecer su perfil y lograr desarrollar las competencias que les permitan gestionar su propia empresa. Asimismo, Fuentelsaz y González (2015) sugieren que para crear nuevos negocios se requiere impulsar el empleo, la innovación y el crecimiento económico, sin embargo, las características económicas y sociales de los jóvenes en el emprendimiento, resultan complejas, debido a que no existe un instrumento analítico, cuantitativo y cualitativo, universalmente aceptado.

Aunado a los problemas anteriores identificados en la población joven, a nivel institucional la problemática identificada es la falta de información sobre un diagnóstico de la situación actual de la intención emprendedora de los estudiantes de los tres Institutos Tecnológicos Superiores Descentralizados pertenecientes al Tecnológico Nacional de México, así como el impacto que puede tener la formación académica, con la intención de fortalecer el Modelo Talento Emprendedor como estrategia institucional de manera focalizada de acuerdo a la información obtenida.

La justificación para realizar la presente investigación es que las IES y también las que ofertan educación superior tecnológica, que anteriormente se centraban en la técnica, hoy juegan una función importante en favor del emprendimiento, debido a que estas, pueden ayudar a los estudiantes y a los egresados a desarrollar las competencias transversales orientadas al desarrollo empresarial y de negocios, que les permitan poner en marcha sus ideas (ANUIES, 2019).

Adicionalmente, como parte del impacto social, el emprendimiento puede impulsar la economía mexicana y ayudar a responder a las necesidades sociales, aunado a que recientemente, se ha observado un crecimiento en el interés de los estudiantes para emprender, sin embargo, de manera general, todavía no existe una cultura sólida del emprendimiento ni mecanismos de apoyo en la educación superior (OECD, 2019).

Por lo anteriormente expuesto, el objetivo de la presente investigación consiste en: evaluar la iniciativa emprendedora de los estudiantes de educación superior tecnológica en México, mediante la Teoría del Comportamiento Planificado, con la finalidad de identificar diferencias significativas respecto a la formación académica.

Asimismo, la principal motivación para desarrollar el proyecto es la integración de un equipo de trabajo de los Cuerpos Académicos de las instituciones participantes, debido a que a partir del diagnóstico se pretende generar información que puede fortalecer los contenidos de las retículas que son parte de la formación académica así como algunas actividades extracurriculares orientadas a fortalecer el emprendimiento, por lo cual el estudio se aborda desde el enfoque de la Teoría del Comportamiento Planificado, dado que se orienta en buscar resultados predictivos que 
permitan establecer una estrategia específica.

El trabajo se estructura de esta introducción, seguida de la revisión de literatura, la metodología, los resultados, la discusión y finalmente las conclusiones.

\section{Revisión de literatura}

La Teoría de Comportamiento Planificado (TCP), es el modelo más utilizado para el estudio del emprendimiento, entre los factores más evaluados se encuentran: autoeficacia, actitud, motivación y normas subjetivas (Valencia, Montoya y Montoya, 2014; Fayolle y Liñán, 2014). Esta teoría, establece como premisa principal un vínculo fuerte entre la intención de actuar y el comportamiento real, de tal forma que para la creación de un nuevo negocio o empresa se requiere una planificación, por lo que representa el tipo de comportamiento planeado ideal para estudiar a través de un modelo de intención (Azjen, 1991).

Asimismo, la teoría se centra en las intenciones de elección de carrera en general y en las intenciones emprendedoras en particular, considerando que la intención de un individuo se influencia por la actitud personal hacia los resultados del comportamiento o hacia las probables consecuencias de su acción; en cuanto a la actitud personal hacia el emprendimiento, se refiere a la actitud hacia el emprendimiento y la asociación con los atributos que dicha persona identifica con el comportamiento emprendedor; también considera la presión percibida de las normas subjetivas que visualiza al entorno que le rodea y por último, comprende el control percibido de su futuro comportamiento (Ajzen, 1991, 2002).

Por lo anterior, la intención emprendedora es el mejor elemento para predecir el comportamiento planeado, especialmente cuando éste es raro, difícil de observar o implica un periodo de tiempo impredecible (Medina, Bolívar y Lemes, 2014). Es así, como la presente investigación se sustenta en dicha teoría, sin embargo, es importante retomar el concepto de emprendedor, del cual en la actualidad no existe un consenso generalizado. Gutiérrez y Amador (2011) mencionan que el emprendedor universitario, es aquella persona con estudios superiores que tiene el conocimiento suficiente y está motivado para iniciar y desarrollar sus sueños, de una manera innovadora, asertiva $\mathrm{y}$ proactiva, llevándolo a producir impactos en la rama productiva elegida.

De igual manera, resulta fundamental retomar la educación empresarial como parte de los programas de estudio, siendo un tema de relevancia para las IES ya que permite desarrollar planes académicos a largo plazo que coadyuven a incentivar la generación de ideas de negocios, así como la creación e incubación de empresas, lo cual puede ser posible a través del desarrollo de las capacidades como la motivación, la innovación y el emprendimiento en los jóvenes y que se desarrollan en la formación profesional (Guevara y Gamboa, 2009; Bellotti, et al., 2014). 
Por su parte, Fuentes y Sánchez (2010) establecen que incentivar la actividad emprendedora en los jóvenes representa un reto, debido a los atributos que debe tener el emprendedor como la originalidad, la aversión al riesgo, la aceptación de responsabilidades, la perseverancia, la autoconfianza, el deseo por aprender y la planificación de metas a largo plazo.

También relacionado al ámbito educativo, Contreras-Velásquez et al (2017) proponen que las universidades diseñen programas de formación en emprendimiento transversal aplicable en todos los programas académicos, adoptando un aprendizaje holístico e integrado que permita que los participantes se beneficien del trabajo colaborativo interdisciplinar. Así también, resulta fundamental considerar que existen factores individuales como rasgos de personalidad, actitudes y el contexto institucional que pueden condicionar las intenciones emprendedoras de los estudiantes de entre 18 y 34 años (Gubik y Farkas, 2016).

Además, resulta importante precisar que en la actualidad el emprendimiento se considera una forma de generar empleo y lograr un modelo de desarrollo socio-económico alternativo que tiene como intención mejorar el bienestar social de individuos que no forman parte del mercado laboral a pesar de contar una carrera universitaria y que pueden encontrar en este una oportunidad de desarrollo profesional (Rangel, Alvarado y Sierra, 2016).

Por otra parte, resulta importante mencionar que uno de los instrumentos para evaluar la intención emprendedora se desarrolla mediante el proyecto GUESSS (Global University Entrepreneurial Spirit Student's Survey) que tiene como propósito el estudio de la intención emprendedora de los estudiantes universitarios para predecir el comportamiento planeado, debido a que la creación de una empresa requiere planificación, por lo tanto, se puede generar información sobre las características individuales que influyen en la intención y en la actividad emprendedora de los estudiantes (Medina et al, 2014).

Cabe destacar que el tema de emprendimiento se ha estudiado en diferentes contextos a nivel internacional. Turker y Sonmez (2009) realizaron una investigación sobre los factores que afectan la intención empresarial de los estudiantes universitarios, aplicando un estudio empírico que probó el modelo de apoyo empresarial (ESM) a una muestra de 300 estudiantes universitarios en Turquía. Los resultados indicaron que se consideraba el impacto de los factores contextuales en la intención empresarial, la cual se genera en función de apoyos educativos, relacionales y estructurales. También Pérez y Sánchez (2019) hicieron un estudio sobre el impacto de la educación en la intención emprendedora de los estudiantes de Economía en una institución de Sevilla-España. Se aplicó el cuestionario a 272 estudiantes y los resultados señalaron que el control del comportamiento percibido había aumentado significativamente tras la 
educación emprendedora; los encuestados manifestaron que sus conocimientos para desarrollar y gestionar exitosamente una empresa se elevaron al recibir educación emprendedora, asimismo, se sentían capaces de resolver prácticamente cualquier problema que se les presentase en el camino hacia el emprendimiento.

En Latinoamérica, Krauss, Bonomo y Volfovicz (2018) desarrollaron una investigación con la finalidad de explorar la intención emprendedora y los factores que incidían en la misma. La muestra se integró por 771 estudiantes de 8 Universidades Latinoamericanas, que por lo menos tenían un curso de Emprendedurismo en sus carrerar del área empresarial o ingeniería. Los resultados consistieron en que la motivación tenía un alto impacto en la intención de emprender, así también, los factores de relacionamiento con la empresa, innovación, motivación y las actitudes personales tuvieron un efecto significativo y positivo sobre la intención emprendedora de los estudiantes universitarios.

En México, Gutiérrez y Amador (2011) compararon el potencial emprendedor de los estudiantes de Contabilidad de un centro universitario de Perú y uno de Guadalajara en México. La principal conclusión fue que en ambas instituciones la iniciativa emprendedora era intermedia, y que las competencias que desarrollaron fueron: la fortaleza ante las dificultades, el aprendizaje, y el afán de logro, y las menos desarrolladas fueron la capacidad de asumir riesgos, la capacidad de tomar decisiones, la flexibilidad, la organización de tiempo y confianza en sí mismos. En Hidalgo, García, Mendoza y Romo (2017) investigaron la intención de elección de la carrera de Ingeniería Civil de los estudiantes de una Institución de Educación Superior Tecnológica, en una muestra de 198 estudiantes. Los resultados determinaron que de los factores que determinaban la iniciativa emprendedora, el mayor puntuado fue la intención emprendedora y el menos puntuado, el control de comportamiento percibido.

\section{Metodología}

El alcance de esta investigación fue descriptivo y de corte transversal. Como unidad de análisis se seleccionaron a los estudiantes inscritos en programas educativos con matrícula de al menos 250 estudiantes, durante el período julio-diciembre 2019, de primero a octavo semestre en la modalidad presencial de los Institutos Tecnológicos Superiores Descentralizados en Hidalgo, México que pertenecen al Tecnológico Nacional de México, obteniéndose una población de 7,165 estudiantes inscritos en 13 programas educativos.

Para el cálculo del tamaño de la muestra de estudio, se aplicó un $95 \%$ de nivel de confianza y un error muestral del $5 \%$, correspondiendo a 365 estudiantes, sin embargo, debido a la posibilidad de aplicar un mayor número de cuestionarios en cada programa educativo se trabajó con una muestra de tamaño mayor, 919 estudiantes, como se 
muestra en la Tabla 1.

El cuestionario fue remitido mediante correo electrónico a los estudiantes de los diferentes programas académicos durante los meses de agosto a octubre de 2019. Para la recolección de datos se aplicó el cuestionario GUESSS (Global University Entrepreneurial Spirit Student's Survey), el cual tiene como propósito comprender la intención de la actividad emprendedora de los estudiantes universitarios (Medina et al, 2014). Este se integra de tres apartados: 1) Información sobre las variables sociodemográficas (institución, programa de estudios y género), 2) Información sobre los antecedentes en materia de emprendimiento, la tradición emprendedora, la formación en materia de emprendimiento, así como la intención de elección de la carrera universitaria con enfoque hacia el pasado y futuro, y 3 ) Medición de cuatro dimensiones: intención emprendedora, actitud personal

Tabla 1.

Datos de la población y muestra por programa educativo

\begin{tabular}{lccc}
\hline Programa Educativo & Población & Porcentaje & Muestra \\
\hline Arquitectura & 758 & $10.32 \%$ & 62 \\
Ingeniería Civil & 419 & $6.08 \%$ & 31 \\
Ingeniería en Electromecánica & 354 & $4.47 \%$ & 46 \\
Ingeniería en Administración & 246 & $2.80 \%$ & 32 \\
Ingeniería en Gestión Empresarial & 986 & $12.66 \%$ & 176 \\
Ingeniería en Industrias Alimentarias & 492 & $6.54 \%$ & 64 \\
Ingeniería en Logística & 643 & $8.34 \%$ & 103 \\
Ingeniería en Mecatrónica & 385 & $4.99 \%$ & 47 \\
Ingeniería en Sistemas Automotrices & 258 & $3.35 \%$ & 39 \\
Ingeniería en Sistemas Computacionales & 811 & $9.65 \%$ & 105 \\
Ingeniería en Tecnologías de la Información y Comunicación & 289 & $2.23 \%$ & 35 \\
Ingeniería Industrial & 1146 & $15.41 \%$ & 136 \\
Licenciatura en Administración & 378 & $5.42 \%$ & 43 \\
Total & 7165 & $100 \%$ & 919 \\
\hline
\end{tabular}

Fuente: Elaboración propia. hacia el emprendimiento, control del comportamiento percibido y normas subjetivas, a partir de las cuales se calculó el índice global de iniciativa emprendedora. La escala de medición usada fue de tipo Likert de 5 puntos, siendo 1: muy en desacuerdo, y 5: muy de acuerdo). En la validación del cuestionario se obtuvo un Alfa de Cronbach de 0.915. Para el análisis estadístico descriptivo de resultados se utilizó el SPSS versión 22.

Para realizar la contrastación de las siguientes hipótesis de investigación planteadas se aplicó la herramienta estadística de análisis factorial ANOVA:

H1: Existen diferencias significativas en la intención emprendedora de los estudiantes respecto a la formación académica.

$\mathrm{H} 2$ : Existen diferencias significativas en la actitud personal hacia el emprendimiento 
de los estudiantes respecto a la formación académica.

H3: Existen diferencias significativas en el control de comportamiento percibido de los estudiantes respecto a la formación académica.

H4: Existen diferencias significativas en las normas subjetivas de los estudiantes respecto a la formación académica.

H5: Existen diferencias significativas en el índice global de iniciativa emprendedora de los estudiantes respecto a la formación académica.

\section{Resultados}

En la Tabla 2, se observa cómo se distribuyó la muestra de estudiantes según el instituto donde estudian, programa que están cursando y sexo. En cuanto a los institutos de los cuales provienen, las participaciones son muy similares. Respecto a los programas de estudio, el mayor número de estudiantes son de: Ingeniería en Gestión Empresarial (19.15\%), Ingeniería en Sistemas Computacionales (11.43\%), Ingeniería Industrial $(14.80 \%) \quad \mathrm{e}$ Ingeniería en Logística (11.21\%). Finalmente, la muestra se integra por $53.10 \%$ de hombres y $46.90 \%$ de mujeres.

\section{Intención de elección de la carrera}

En la Tabla 3, se muestran los resultados del alcance descriptivo del estudio, respecto a las preguntas relacionadas con la intención de elección

Tabla 2.

Datos de la muestra por variables sociodemográficas

\begin{tabular}{|c|c|c|c|}
\hline Variable & Descripción & Frecuencia & Porcentaje \\
\hline \multirow{4}{*}{ 总 } & ITESA & 317 & 34.49 \\
\hline & ITESHU & 290 & 31.56 \\
\hline & ITSOEH & 312 & 33.95 \\
\hline & Total & 919 & 100.00 \\
\hline \multirow{14}{*}{ 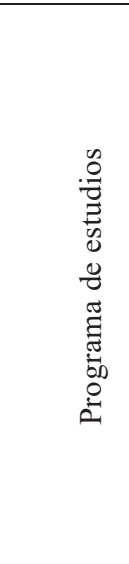 } & Arquitectura & 62 & 6.75 \\
\hline & Ingeniería Civil & 31 & 3.37 \\
\hline & Ingeniería en Electromecánica & 46 & 5.01 \\
\hline & Ingeniería en Administración & 32 & 3.48 \\
\hline & Ingeniería en Gestión Empresarial & 176 & 19.15 \\
\hline & Ingeniería en Industrias Alimentarias & 64 & 6.96 \\
\hline & Ingeniería en Logística & 103 & 11.21 \\
\hline & Ingeniería en Mecatrónica & 47 & 5.11 \\
\hline & Ingeniería en Sistemas Automotrices & 39 & 4.24 \\
\hline & Ingeniería en Sistemas Computacionales & 105 & 11.43 \\
\hline & Ingeniería en Tecnologías de la Información y Comunicación & 35 & 3.81 \\
\hline & Ingeniería Industrial & 136 & 14.80 \\
\hline & Licenciatura en Administración & 43 & 4.68 \\
\hline & Total & 919 & 100 \\
\hline \multirow{3}{*}{ 号 } & Masculino & 488 & 53.10 \\
\hline & Femenino & 431 & 46.90 \\
\hline & Total & 919 & 100 \\
\hline
\end{tabular}

Fuente: Elaboración propia. 
de la carrera al inicio y término, y cinco años después de concluida. Estos indican que, de manera general, la opción de ser fundador de mi propia empresa representa el mayor porcentaje en los tres momentos, incrementándose en $10.2 \%$ del momento de la elección de carrera a cinco años después de terminar los estudios. De igual forma se observa que la alternativa de ser empleado disminuye en $13.4 \%$.

Por otra parte, en la Tabla 4 se muestran los resultados de las puntuaciones medias de las cuatro dimensiones que integran el Índice Global de Iniciativa Emprendedora con un resultado de (4.1121), que se refiere a que la mayoría de los estudiantes están de acuerdo en emprender en algún momento. En cuanto a las dimensiones sus puntuaciones registraron el siguiente orden: intención emprendedora (4.2410), actitud personal hacia el emprendimiento (4.1979), normas subjetivas (4.1121) y control de comportamiento percibido (3.9032), lo cual indica que el factor que más se presenta en los estudiantes es la intención emprendedora de iniciar o desarrollar una idea de negocio en el

Tabla 3

Intención de la elección de carrera (\%)

\begin{tabular}{lcccc}
\hline \multicolumn{1}{c}{ Iniciativa Emprendedora } & $\begin{array}{c}\text { Ser } \\
\text { empleado }\end{array}$ & $\begin{array}{c}\text { Ser fundador } \\
\text { de mi propia } \\
\text { empresa }\end{array}$ & $\begin{array}{c}\text { Ser } \\
\text { sucesor } \\
\text { de una } \\
\text { empresa }\end{array}$ & $\begin{array}{c}\text { Otro, no } \\
\text { lo sé } \\
\text { todavía }\end{array}$ \\
\hline $\begin{array}{l}\text { 1.Como estudiante ¿Cuál fue su intención al elegir } \\
\text { su carrera? }\end{array}$ & 21.9 & 64.7 & 2.8 & 10.6 \\
$\begin{array}{l}\text { 2. ¿Cuál es su intención de elegir su carrera, al } \\
\text { terminar sus estudios? }\end{array}$ & 18.4 & 65.4 & 6.5 & 9.7 \\
$\begin{array}{l}\text { 3. ¿Cuál es su intención al elegir su carrera, cinco } \\
\text { años después de terminar sus estudios? }\end{array}$ & 8.5 & 74.9 & 8.2 & 8.4 \\
\hline
\end{tabular}

Fuente: Elaboración propia.

Tabla 4.

Dimensiones de la Iniciativa Emprendedora

\begin{tabular}{|c|c|c|c|c|c|c|c|}
\hline \multirow[b]{2}{*}{ Dimensión } & \multirow[b]{2}{*}{$\mathrm{N}$} & \multicolumn{6}{|c|}{$\begin{array}{l}95 \% \text { del intervalo de } \\
\text { confianza para la media }\end{array}$} \\
\hline & & Media & $\begin{array}{l}\text { Desviación } \\
\text { Estándar }\end{array}$ & $\begin{array}{l}\text { Límite } \\
\text { inferior }\end{array}$ & $\begin{array}{l}\text { Límite } \\
\text { superior }\end{array}$ & Mínimo & Máximo \\
\hline Intención Emprendedora & 919 & 4.2410 & 0.81789 & 4.1881 & 4.2940 & 1.0000 & 5.0000 \\
\hline $\begin{array}{l}\text { Actitud Personal hacia el } \\
\text { Emprendimiento }\end{array}$ & 919 & 4.1979 & 0.77012 & 4.1481 & 4.2478 & 1.0000 & 5.0000 \\
\hline $\begin{array}{l}\text { Control de Comportamiento } \\
\text { Percibido }\end{array}$ & 919 & 3.9032 & 0.78096 & 3.8526 & 3.9537 & 1.0000 & 5.0000 \\
\hline Normas Subjetivas & 919 & 4.1121 & 0.80838 & 4.0597 & 4.1644 & 1.0000 & 5.0000 \\
\hline $\begin{array}{l}\text { Índice Global de Iniciativa } \\
\text { Emprendedora }\end{array}$ & 919 & 4.1121 & 0.69244 & 4.0672 & 4.1569 & 1.0000 & 5.0000 \\
\hline
\end{tabular}

Fuente: Elaboración propia. 
futuro. Mientras que la puntuación más baja corresponde al control de comportamiento percibido, relacionado con el grado que asume el estudiante para desarrollar el comportamiento específico de emprender, es decir, que están de acuerdo en emprender, ya sea al término de sus estudios o después.

Análisis de varianza factorial ANOVA

Para realizar el contraste de las hipótesis, se aplicó la prueba estadística de ANOVA, con el propósito de identificar si existen diferencias estadísticamente significativas entre los grupos analizados por programa educativo para las dimensiones de estudio y de manera general.

Tabla 5.

Prueba ANOVA

\begin{tabular}{llc}
\hline Dimensión & $\mathrm{F}$ & Sig. \\
\hline Intención Emprendedora & 1.465 & 0.132 \\
$\begin{array}{l}\text { Actitud Personal hacia el } \\
\text { Emprendimiento }\end{array}$ & 1.804 & $0.043^{*}$ \\
$\begin{array}{l}\text { Control de Comportamiento } \\
\text { Percibido }\end{array}$ & 1.626 & 0.079 \\
$\begin{array}{l}\text { Normas Subjetivas } \\
\text { Índice Global de Iniciativa }\end{array}$ & 2.068 & $0.017^{*}$ \\
Emprendedora & & \\
\hline $\begin{array}{l}\text { Nota: } \mathrm{P}<0.05, * * \mathrm{p}<0.01 \\
\text { Fuente: Elaboración propia. }\end{array}$ & & \\
\end{tabular}

En la Tabla 5 se demuestra que hay suficiente evidencia estadística para no rechazar las siguientes hipótesis:

$\mathrm{H} 2$ : Existen diferencias significativas en la actitud personal hacia el emprendimiento de los estudiantes respecto a la formación académica.

H4: Existen diferencias significativas en las normas subjetivas de los estudiantes respecto a la formación académica.

H5: Existen diferencias significativas en el índice global de iniciativa emprendedora de los estudiantes respecto a la formación académica.

Y, en consecuencia, se rechazaron las siguientes:

$\mathrm{H} 1$ : Existen diferencias significativas en la intención emprendedora de los estudiantes respecto a la formación académica.

H3: Existen diferencias significativas en el control de comportamiento percibido de los estudiantes respecto a la formación académica.

\section{Diagrama de árbol}

En la Figura 1 se presenta el diagrama de árbol, con el cual se generó la clasificación de los casos de estudio en grupos, considerando como variable dependiente al Índice global de iniciativa emprendedora y como independiente el programa de estudios. La integración de los nodos del diagrama de árbol se realizó mediante el método Chaid, con el cual se segmentaron y estratificaron de acuerdo con las puntuaciones medias obtenidas en la variable dependiente, por lo que partir de las relaciones, se generó una representación gráfica que representa los sucesos mediante la fusión de categorías en tres nodos, identificándose que el segundo nodo tiene menor iniciativa emprendedora, mientras que el tercer 
nodo reflejó una mayor intención de emprendimiento (4.320). El nodo 3 representa el $22.6 \%$ de los estudiantes, quienes estudian los programas de Ingeniería en Administración e Ingeniería en Gestión Empresarial.

Los resultados de esta investigación concuerdan con los obtenidos en España y Colombia, los cuales presentaron incremento de la intención emprendedora de los estudiantes cinco años después de concluir sus estudios, lo cual puede ocurrir debido a que al término de los estudios no han obtenido el desarrollo de habilidades que les permitan asumir el riesgo (Medina et al, 2014; Álvarez, Martins y López, 2017).

En cuanto a las puntuaciones medias de las dimensiones evaluadas en el cuestionario GUESSS, los resultados fueron similares a los que obtuvieron García et al. (2017), donde las dos dimensiones con la mayor media son la intención emprendedora y la actitud personal hacia el emprendimiento, que se refieren a la intención de crear un negocio y los atributos que se identifican en el comportamiento emprendedor. Asimismo, los resultados menores en las medias de control de comportamiento percibido y normas subjetivas, sugieren el trabajo con los estudiantes en contenidos relacionados con la tolerancia al riesgo e inclusión de herramientas que puedan fortalecer las consecuencias de asumir determinado comportamiento, así como en las normas subjetivas el manejo de la información de las opiniones negativas

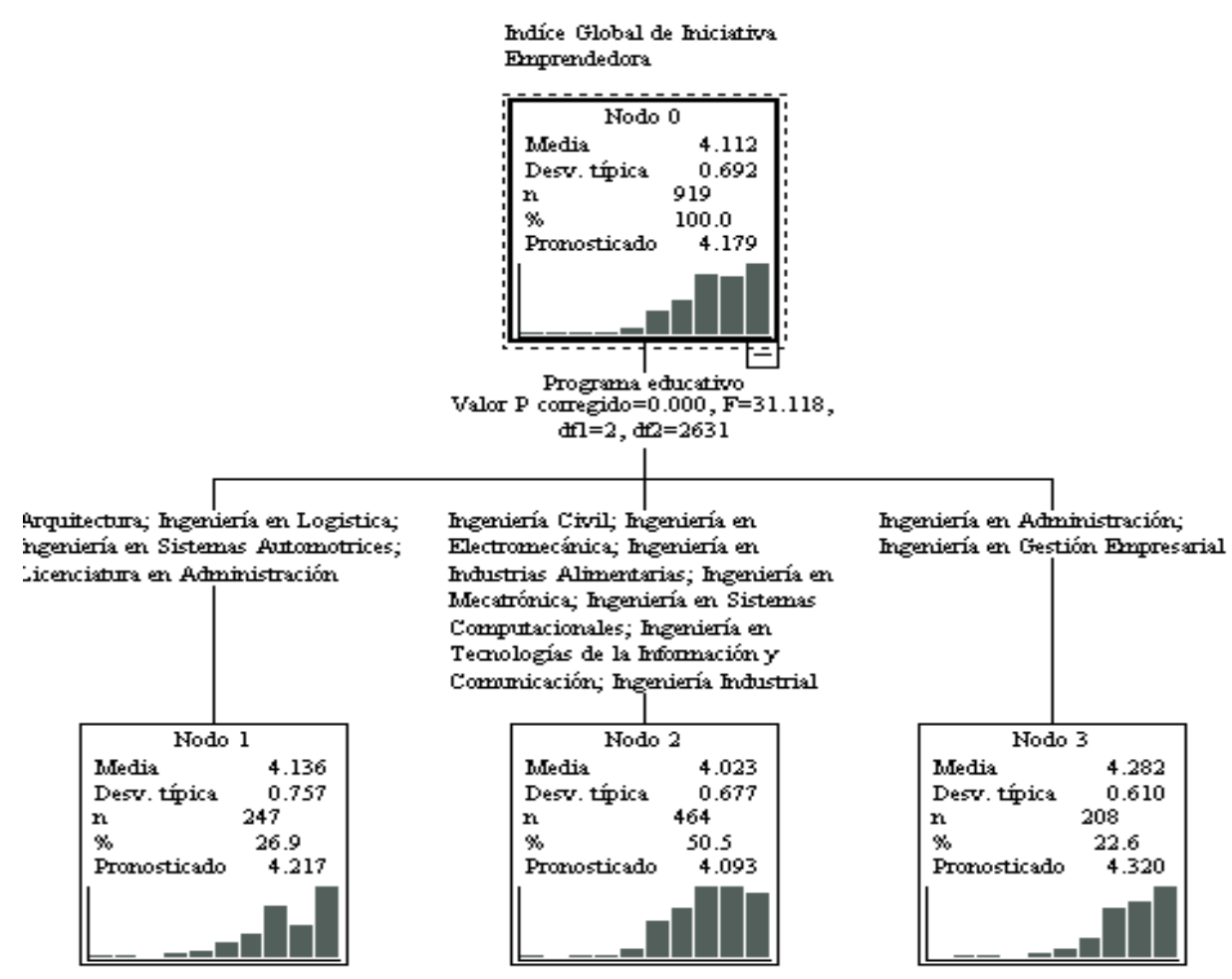

Figura 1. Diagrama de árbol de la Iniciativa Emprendedora.

Fuente: Elaboración propia. 
que pudieran tener familiares o amigos.

Respecto al índice global de iniciativa emprendedora, se observó un área de oportunidad para los programas de estudio del nodo 2, que en su retícula no contienen asignaturas orientadas al emprendimiento, como son los programas de: Ingeniería Civil, Ingeniería Electromecánica, Ingeniería en Sistemas Computacionales, Ingeniería en Mecatrónica, Ingeniería en Industrias Alimentarias, Ingeniería en Tecnologías de la Información y Comunicación, Ingeniería Industrial, de tal forma que puedan fortalecer las metodologías de enseñanza-aprendizaje que pueden incidir en la intención emprendedora y capacidades empresariales (Liñan y Fayolle, 2015). De igual forma se sugiere retomar la propuesta de trabajo de la auto-eficacia empresarial mediante el aprendizaje pasado como estrategia para fortalecer la intención de emprender (Bell, Dearman y Wilbanks, 2015).

Los resultados del nodo 3 , que identificaron una puntuación mayor en la intención de emprender para los estudiantes de Ingeniería en Administración e Ingeniería en Gestión Empresarial, considerando que la variable independiente es la formación académica, en este grupo se integran programas educativos que en su retícula contienen algunas asignaturas relacionadas con los negocios, administración, finanzas, logística, producción, mercadotecnia, recursos humanos, entre otras que pueden contribuir a generar competencias orientadas a dicha intención como parte de la definición del perfil de egreso. De manera general, se sugiere también establecer para todos los programas de estudio el desarrollo de sesiones con emprendedores exitosos, con la intención de que los estudiantes conozcan y se motiven con vivencias reales (CaroGonzález, Romero-Benabent, H. A., y Sánchez-Torné, 2017).

Para trabajar en la mejora del control de comportamiento percibido y las normas subjetivas, se recomienda la inclusión de temas relacionados con manejo de la seguridad en sí mismos, competencias y características emprendedoras (Karabulut y Dogan, 2018).

El resultado sobre la intención emprendedora de los estudiantes de educación superior tecnológica, no es muy diferente a los resultados de la intención emprendedora universitaria a nivel internacional, lo cual ante la limitada oferta laboral, puede ser una oportunidad para que las IES contribuyan a fortalecer el emprendimiento y en el diseño de estrategias que permitan disminuir los factores que pueden desalentar a quienes tienen como opción de elección de carrera el crear su propio negocio. Finalmente, es necesario mencionar lo expuesto por Murillo y Montaño (2018) que como resultado de su investigación concluyeron que los egresados de las instituciones educativas públicas tienen condiciones más adversas en términos de salario, estabilidad laboral y puestos desempeñados, a diferencia de los egresados de instituciones privadas, considerando que puede ser una alternativa para los futuros profesionistas. 


\section{Conclusiones}

El emprendimiento ha generado un fuerte interés como potencial generador de opción de desarrollo y crecimiento profesional, por lo cual, es indiscutible el papel que desempeñan las IES, al ser la instancia en la cual los estudiantes y futuros profesionistas desarrollan las competencias y habilidades necesarias que los ayuden a generar la actividad emprendedora.

Por lo anterior, en la presente investigación se logró el objetivo de evaluar la iniciativa emprendedora de los estudiantes de educación superior tecnológica, mediante la Teoría del Comportamiento Planificado con la finalidad de identificar diferencias significativas respecto a la formación académica. Asimismo, a partir del análisis descriptivo se pudo concluir que la intención emprendedora de los estudiantes se incrementa considerablemente cinco años después de concluir los estudios. Además, que las participaciones de las dimensiones del índice global de iniciativa emprendedora se presentaron en el siguiente orden: iniciativa emprendedora, actitud personal hacia el emprendimiento, normas subjetivas y control de comportamiento percibido. Como resultado de la prueba ANOVA, se concluyó que existen diferencias estadísticamente significativas en las puntuaciones medias de la actitud personal hacia el emprendimiento, en las normas subjetivas y en el índice global de iniciativa emprendedora de acuerdo a la formación académica de los encuestados.

Por lo tanto, las IES deben trabajar en el desarrollo y fortalecimiento de programas de estudios que integren el emprendimiento en el currículo, como parte de las competencias transversales que puedan fortalecer la formación de los estudiantes, de tal forma que consideren el emprendimiento como una opción laboral, y se consolide la oportunidad de iniciar y gestionar con éxito un negocio (OECD, 2019; ANUIES, 2019). Al respecto Sánchez, Hernández y Jiménez (2016) señalan que las IES deben fortalecer el desarrollo de modelos de enseñanza orientados al emprendimiento, en los cuales los estudiantes encuentren espacios para el desarrollo de ideas y la consejería adecuada que les ayude a identificar la forma en la que se pueden obtener recursos económicos que sirvan como capital semilla. Lo anterior, fortalece los perfiles de egreso que establecen en algún apartado el emprendimiento, lo cual ante la situación de empleo actual que se caracteriza por limitadas oportunidades, puede favorecer la dinámica económica del estado y del país, generando alternativas para que los futuros egresados puedan crear, desarrollar o consolidar una empresa.

Así también, es indiscutible el fortalecimiento de la educación empresarial, bajo la premisa de que existe interés de los estudiantes de emprender en un futuro, por lo cual es fundamental mejorar continuamente los programas de emprendimiento con el propósito de despertar las emociones y transformar mentalidades en realidades de desarrollo de negocios, lo que implica trabajar con los programas de estudio de las carreras universitarias (Souitaris, Zerbinati y Al-Laham, 2007; Kakouris, 2015).

Este estudio generó información que 
puede servir de referencia para que a partir de los resultados obtenidos, se puedan definir estrategias claras sobre los requerimientos desde el punto de vista educativo para fortalecer la formación de los futuros profesionales orientados al emprendimiento. La principal limitación que presentó la investigación fue que solamente se aplicó a una muestra de tres instituciones, por lo cual resultaría interesante replicar el estudio en otros campus del Tecnológico Nacional de México. Como futura línea de investigación, se sugiere realizar el análisis con variables como el género y los antecedentes en emprendimiento, así como mediante la evaluación del impacto del Modelo Talento Emprendedor y la integración del enfoque cualitativo.

\section{Agradecimientos}

Se agradece al Tecnológico Nacional de México y al Gobierno del Estado de Hidalgo por el financiamiento del proyecto, al Instituto Tecnológico Superior del Oriente del Estado de Hidalgo (ITESA), Instituto Tecnológico Superior del Occidente del Estado de Hidalgo (ITSOEH) e Instituto Tecnológico Superior de Huichapan (ITESHU) por las facilidades otorgadas para la ejecución del proyecto, así como a los estudiantes: Alfredo Castillo Olvera, Diana Lizbeth Ortega Ugalde y Donagy Ramírez Vera por su colaboración en la ejecución del proyecto.

\section{Referencias}

Álvarez, C., Martins, I., y López, T. (2017). El espiritu emprendedor de los estudiantes en Colombia Resultados del Proyecto Guesss Colombia 2016. Medellín: Universidad EAFIT.

Ajzen, I. (1991). The theory of planned behavior. Organizational behavior and human decision processes, 50(2),179-211. doi: 10.1016/0749-5978(91)90020-T

Ajzen, I. (2002). Perceived Behavioral Control, Self-efficacy, Locus of Control, and the Theory of Planned Behavior. Journal of Applied Social Psychology, 32(4), 665-683. doi: 10.1111/j.1559-1816.2002. tb00236.x

Asociación Nacional de Universidades e Instituciones de Educación Superior- ANUIES (2019). Las Instituciones de Educación Superior, promotoras del emprendimiento: Jaime Valls Esponda. Recuperado de http://www.anuies.mx/noticias/las-institu ciones-de-educacin-superior-promotorasdel-emprendimiento

Bell, J.R., Dearman, D., y Wilbanks, J.E. (2015). Evaluating the effects of a problem-based learning business planning course on student entrepreneurial intentions. Journal of Entrepreneurship Education, 18(1), 169-182.

Bellotti, F., Berta, R., De Gloria, A., Lavagnino, E., Antonaci, A., Dagnino, F., Ott, M., Romero, M., Usar, M., y Mayer, I. S. (2014). Serious games and the development of an entrepreneurial mindset in higher education engineering students. Entertainment Computing, 5(4), 357-366. doi: 10.1016/j.entcom.2014.07.003

Bucardo, M. A., Saavedra, M. y Camarena, M. (2015). Hacia una comprensión de los conceptos de emprendedores y empresarios. Suma de Negocios, 6(13), 98-107. doi: 10.1016/j.sumneg.2015.08.009

Caro-González, F. J., Romero-Benabent, H., y Sánchez-Torné, I. (2017). The influence of gender on the entrepreneurial 
intentions of journalism students. Intangible Capital, 13(2), 430-478. doi:/10.3926/ic.927

Contreras-Velásquez, J.C. Wilches-Duran, S. Y., Graterol-Rivas, M. y Bautista-Sandoval, M. (2017). Educación Superior y la Formación en Emprendimiento Interdisciplinario: Un Caso de Estudio. Formación Universitaria, 10(3), 11-20. doi: $10.4067 / \mathrm{S} 0718-50062017000300003$

De Domingo-Soler, C., Naveda-Jácome,M., Rodríguez-Proaño, M.A., y MuñozPumagualle, M.G.(2020). Juventud, academia y empleo. Análisis de una desconexión. Podium, 37, 129-146. doi:10.31095/podium.2020.37.9

Duval-Couetil, N., Gotch, C. y Yi, S. (2014). The characteristics and motivations of contemporary entrepreneurship students. Journal of Education for Business, 89(4), 441-449. doi: 10.1080/08832323.2014. 933156

Fayolle, A. y Liñán, F. (2014). The Future of Research on Entrepreneurial Intentions. Journal of Business Research, 67(5), 663-666. Doi: 10.1016/j.jbusres.2013.11.024

Fuentes, F., y Sánchez, S. (2010). Análisis del perfil emprendedor: una perspectiva de género. Estudios de Economía Aplicada, 28(3), 1-28.

Fuentelsaz, L., y González, C. (2015). El fracaso emprendedor a través de las instituciones y la calidad del emprendimiento. Universia Business Review, 47, 64-81.

García, Y., Mendoza, I., y Romo, L. (2017). Relación Entre La Intención De La Elección De La Carrera De Ingeniería Civil y La Iniciativa Emprendedora De Los Estudiantes De Una Institución De Educación Superior Tecnológica En Hidalgo, México. European Scientific Journal, 13(34), 101-118. doi:10.19044/ esj.2017.v13n34p101
Gubik, A., y Farkas, S. (2016). Student Entrepreneurship in Hungary: Selected Results Based on Guesss Survey. Entrepreneurial Business and Economics Review, 4(4), 123-139.

Guevara, M., y Gamboa, O. (2009). Experiencias de incorporación de emprendedurismo en Instituciones de Educación Superior. ICAP-Revista Centroamericana de Administración Pública, (56-57), 171-182.

Gutiérrez, A. M., y Amador, M. E. (2011). El potencial emprendedor en los estudiantes de la carrera de Contabilidad de las universidades San Marcos de Perú y Guadalajara de México, Centro Universitario de los Altos, un análisis comparativo. Quipukamayoc, 19(36), 63-82. doi: 10.15381/quipu.v19i36.6497

Hernández, C.A., y Sánchez, S. (2017). La educación empresarial: un acercamiento desde los estudiantes universitarios en dos instituciones de educación superior. Innovación Educativa, 17(75), 81-102.

Karabulut, E. O., y Dogan, P. K. (2018). Investigation of Entrepreneurship Trends and General Competency Levels of University Students Studying at Faculty of Sports Sciences. Journal of Education and Training Studies, 6(4), 212-220.

Kakouris, A. (2015). Entrepreneurship pedagogies in lifelong learning: Emergence of criticality? Learning, Culture and Social Interaction, 6, 87-97. doi:10.1016/j.lcsi. 2015.04.004

Krauss, C., Bonomo, A., y Volfovicz, R. (2018). Modelo predictivo de la intención emprendedora universitaria en Latinoamérica. Journal of Technology Management \& Innovation, 13(4), 84-93. doi: $10.4067 / \mathrm{S} 0718-27242018000400084$

Liñán, F., y Fayolle, A. (2015). A systematic literature review on entrepreneurial intentions: Citation, thematic analyses, 
and research agenda. International Entrepreneurship and Management Journal, 11, 907-933. doi: 10.1007/s11 365-015-0356-5.

Medina, P., Bolívar, A., y Lemes, A.I. (2014). Un paso más en la investigación de la intención emprendedora del estudiante universitario: GUEESS. Revista de Estudios Empresariales. Segunda época, (2), 63-80. doi:10.17561/ree

Murillo, F., y Montaño, P. Y. (2018). Condiciones laborales de egresados de Instituciones de Educación Superior en México. Revista Electrónica de Investigación Educativa, 20(3), 56-68. doi: 10.24320/redie.2018. 20.3.1644

Organización para la Cooperación y el Desarrollo Económicos- OECD. (2019). Educación Superior en México. Resultados y relevancia para el mercado laboral. Paris: OECD Publishing. Recuperado de: https://www.oecd.org/centrodemexico/m edios/educacion_superior_en_mexico.pdf

Rangel, O., Alvarado, M., y Sierra, E. (2016). El perfil emprendedor como catalizador de la innovación en los jóvenes del Departamento de la Guajira. Omnia, 22(3),71-86.

Sánchez, S., Hernández, C.A., y Jiménez, M. (2016). Análisis de la percepción sobre iniciativa empresarial y el espíritu emprendedor en estudiantes de un tecnológico federal. Acta Universitaria, 26(6), 70-82. doi: 10.15174/au.2016.1016

Pérez, M., y Sánchez, I. (2019). El impacto de la educación en la intención emprendedora de los estudiantes del grado de Economía. Revista de Estudios Empresariales. Segunda época, (1), 22-40. doi: 10.17561/ree.v2019n1.2

Secretaría de Economía. (2017). Emprendedores. Recuperado de http://www.2006- 2012. economia.gob.mx/mexico-emprende/em presas/emprendedor

Souitaris, V., Zerbinati, S., y Al-Laham, A. (2007). Do entrepreneurship programmes raise entrepreneurial intention of science and engineering students? The effect of learning, inspiration and resources. Journal of Business Venturing, 22(4), 566-591. doi: 10.1016/j.jbusvent.2006. 05.002

Turker, D., y Sonmez, S. (2009). ¿Qué factores afectan la intención empresarial de los estudiantes universitarios?. Journal of European Industrial Training, 33(2), 142-159. doi: 10.1108/03090590910939049.

Valencia, A., Montoya, I., y Montoya, A. (2016). Intención emprendedora en estudiantes universitarios: Un estudio bibliométrico. Intangible Capital, 12(4), 881-922. doi: 10.3926/ic. 730 